PROCEEDINGS OF THE

AMERICAN MATHEMATICAL SOCIETY

Volume 138, Number 4, April 2010, Pages 1231-1242

S 0002-9939(09)10179-X

Article electronically published on December 1, 2009

\title{
VARIATIONS ON A QUESTION OF LARSEN AND LUNTS
}

\author{
JULIEN SEBAG
}

(Communicated by Ted Chinburg)

\begin{abstract}
Let $k$ be a field of characteristic zero. Let $X$ and $X^{\prime}$ be two $k$-schemes of finite type having the same class in the Grothendieck ring of varieties. Is it true that $X$ and $X^{\prime}$ are piecewise isomorphic? This question, originally asked by Larsen and Lunts, and some of its consequences constitute the subject of this article.
\end{abstract}

\section{INTRODUCTION}

Assume that $k$ is a field. Let us define two notions which are central in the present article:

a) Let $X, X^{\prime}$ be two $k$-schemes of finite type. We say that $X$ and $X^{\prime}$ are piecewise isomorphic if there exist a finite partition of $X$ into locally closed parts $\left(X_{i}\right)_{i \in I}$, a finite partition of $X^{\prime}$ into locally closed parts $\left(X_{i}^{\prime}\right)_{i \in I}$, and a bijection $\sigma: I \rightarrow I$ such that, for each $i \in I,\left(X_{i}\right)_{\text {red }}$ is isomorphic to $\left(X_{\sigma(i)}^{\prime}\right)_{\text {red }}$ (as $k$-schemes).

b) Let $\mathbb{Z}\left[\operatorname{Var}_{k}\right]$ be the free abelian group generated by the isomorphism classes of all the $k$-schemes of finite type. We denote by $\{X\}$ the class in $\mathbb{Z}\left[V a r_{k}\right]$ of $X$. Let $K_{0}\left(V a r_{k}\right)$ be the quotient of $\mathbb{Z}\left[V a r_{k}\right]$ by the subgroup generated by the elements of the form $\{X\}-\{Y\}-\{X \backslash Y\}$, for all the $k$-schemes of finite type $X$ and all the closed subschemes $Y$ of $X$. In this group, we denote by $[X]$ the class of $X$. The fibered product over $k$ endows $K_{0}\left(\operatorname{Var}_{k}\right)$ with a ring structure, by setting $\left[X \times_{k} X^{\prime}\right]=[X] \cdot\left[X^{\prime}\right]$, for all the $k$-schemes of finite type $X$ and $X^{\prime}$. For this structure, the neutral element is $1=[\operatorname{Spec}(k)]$. This ring is called the Grothendieck ring of varieties. We denote by $\mathbb{L}:=\left[\mathbb{A}_{k}^{1}\right]$ the class in $K_{0}\left(V a r_{k}\right)$ of the affine line $\mathbb{A}_{k}^{1}$ over $k$.

Such an object, and more generally such notions, is an important piece of the theory of motivic integration (see, for example, [8]). Thus a good understanding of the Grothendieck ring of varieties, which is naturally and closely related to cohomological theories and motives, seems to us to be an interesting and important question of algebraic geometry. Yet little information is known about it. In [5], Larsen and Lunts ask the following question. Up to now, this question remains open in the general case (see Remark 3.6).

Question LL. Let $k$ be a field of characteristic zero. Let $X$ and $X^{\prime}$ be two $k$ schemes of finite type such that $[X]=\left[X^{\prime}\right]$ in $K_{0}\left(\operatorname{Var}_{k}\right)$. Is it true that $X$ and $X^{\prime}$ are piecewise isomorphic?

Received by the editors March 2, 2009, and, in revised form, August 25, 2009.

2000 Mathematics Subject Classification. Primary 14E05; Secondary 14E07, 14R10. 
Question LL (and so the geometric understanding of the Grothendieck ring of varieties) is the subject of the present article. The heart of this article is contained in 4 . In this section, we motivate such a question by setting, and studying, two new challenging problems (see Questions $\mathrm{R}$ and $\mathrm{Cr}$ ), linked to question LL. More precisely, if question LL admits a positive answer, our questions $\mathrm{R}$ and $\mathrm{Cr}$ admit a positive answer too. Furthermore, we give partial (i.e., in dimensions 1 or 2), but unconditional, solutions to each of these two problems (see Propositions 4.2 and 4.6). In 33, we complete, for $k$-varieties of dimension one or two and in the non- "smooth and projective" context (see Theorem 3.3), the previous answers to question LL, which we obtained in [7. The results of the present article use the birational cancellation techniques, we introduced in [7, and we recall in $\$ 2$ (see Theorem 2.31). An important class of varieties still gets away from our strategy of cancellation: the uniruled $k$-varieties. In our mind, giving an answer to question LL for uniruled varieties is a deep and difficult problem. To justify this last point of view, we exhibit an original link between question LL and that of determining if $\mathbb{L}$ is (or is not) a zero divisor in $K_{0}\left(\operatorname{Var}_{k}\right)$. This last problem has remained, up to now, totally open, but is considered to be important in the "world" of motivic integration. We show that a positive answer to question LL has an impact on the description of the kernel of the multiplication by $\mathbb{L}$ (see Proposition 4.9). Besides, we prove that "curves" are not in the kernel of this multiplication if and only if the (uni)ruled surfaces verify the condition of question LL (see Theorem 3.3 and Remark 4.11).

Finally, we remark that all the results in $\$ 3$ and $\$ 4$ are original, and those of $\$ 3$ cannot be deduced from our previous ones (see Remark 3.6). All these results underline the fact that the question of the existence of rational curves on varieties, a main question in birational geometry, also seems crucial in order to understand the Grothendieck ring of varieties.

\section{Notation AND teChNiCAl Lemmas}

Notation. a) In this article, $k$ is a field of characteristic zero. A $k$-variety $X$ denotes a reduced $k$-scheme of finite type and separated. We say that two $k$ schemes $X$ and $X^{\prime}$ of finite type are stably birational if there exist two integers $m$, $m^{\prime} \in \mathbf{N}$ such that $X \times_{k} \mathbb{P}_{k}^{m}$ is birational to $X^{\prime} \times_{k} \mathbb{P}_{k}^{m^{\prime}}$. An integral $k$-variety $X$ of dimension $d$ is uniruled if there exists an integral $k$-variety $Y$ of dimension $d-1$ and a dominant rational map $Y \times_{k} \mathbb{P}_{k}^{1} \rightarrow X$. A $k$-curve (surface) is a $k$-variety of dimension 1 (dimension 2).

b) Let $k$ be an algebraically closed field of characteristic zero. Recall that if $X$ is a smooth proper $k$-variety, its Poincaré polynomial is defined by $P_{X}(u)=$ $\sum_{n=0}^{2 \operatorname{dim} X} \operatorname{dim}_{k} H_{\mathrm{dR}}^{n}(X) u^{n} \in \mathbb{Z}[u]$. Let us set $b_{n}(X):=\operatorname{dim}_{k} H_{\mathrm{dR}}^{n}(X)$. One knows (see, for example, [2]) that the assignment of Poincaré polynomials induces a ring morphism $K_{0}\left(\operatorname{Var}_{k}\right) \rightarrow \mathbb{Z}[u]$, sending a smooth proper $k$-variety $X$ to its Poincaré polynomial $P_{X}(u)$.

c) Let $\mathfrak{a} \in K_{0}\left(\operatorname{Var}_{k}\right)$. There exists a finite number of connected smooth projective $k$-varieties $X_{i}, i \in I$, and $Y_{j}, j \in J$, such that $\mathfrak{a}=\sum_{i \in I}\left[X_{i}\right]-\sum_{j \in J}\left[Y_{j}\right]$. We call the dimension of $\mathfrak{a}$ the smallest integer $n$ such that all the $X_{i}$ and all the $Y_{j}$ are of dimension less than or equal to $n$. 
Technical lemmas. One can show:

Lemma 2.1. Let $k$ be a field of characteristic zero. Let $X, X^{\prime}$ be two k-varieties. Assume that one of the following properties is true:

a) $X$ is piecewise isomorphic to $X^{\prime}$;

b) $k$ is algebraically closed and $[X]=\left[X^{\prime}\right]$ in $K_{0}\left(\operatorname{Var}_{k}\right)$.

Then $X$ and $X^{\prime}$ have the same dimension and the same number of irreducible components of maximal dimension.

Proof. Remark that the dimension of an irreducible $k$-variety $S$ is also the transcendence degree (over $k$ ) of its functions field. The assertion for a) then follows directly from the definition of a piecewise isomorphism. For b), conclude by [7, Corollary 5].

We denote by $\mathbf{Z}[\mathfrak{s} \mathfrak{b}]$ the free abelian group generated by the stable birational equivalence classes of connected smooth projective $k$-varieties.

Theorem 2.2 (Larsen-Lunts, [5]; see also [1]). Let $k$ be a field of characteristic zero. There exists a unique group morphism $S B: K_{0}\left(\operatorname{Var}_{k}\right) \rightarrow \mathbb{Z}[\mathfrak{s} \mathfrak{b}]$, sending the class of a connected smooth proper $k$-variety to its stable birational equivalence class. The kernel of $S B$ is the ideal generated by $\mathbb{L}$.

Theorem 2.3 (Liu-Sebag, [7). Let $k$ be a field of characteristic zero. Let $X, X^{\prime}$ be integral $k$-varieties of the same dimension. Assume that $X$ and $X^{\prime}$ are stably birational and that $X$ is non-uniruled. Then $X^{\prime}$ is non-uniruled. Furthermore, $X$ and $X^{\prime}$ are birational.

As an application of the two previous theorems, we have shown in 7, Proposition 5, Proposition 6]:

Theorem 2.4 (Liu-Sebag, 7]). a) Let $k$ be a field of characteristic zero. Let $X$, $X^{\prime}$ be zero-dimensional $k$-varieties such that $[X]=\left[X^{\prime}\right]$ in $K_{0}\left(\operatorname{Var}_{k}\right)$. Then $X$ is isomorphic to $X^{\prime}$. b) Let $k$ be an algebraically closed field of characteristic zero. Let $X$ and $X^{\prime}$ be $k$-varieties such that $\operatorname{dim} X \leq 1$ and $[X]=\left[X^{\prime}\right]$ in $K_{0}\left(\operatorname{Var}_{k}\right)$. Then $X$ is piecewise isomorphic to $X^{\prime}$.

The following remark will be used throughout the article. We recall here some elementary results for the convenience of the reader.

Remark 2.5. a) In Lemma 2.1, under assumption a), we also see that the irreducible components of maximal dimension of $X$ and those of $X^{\prime}$ are pairwise birational. Indeed, by the definition of a piecewise isomorphism, there is a bijection $\sigma$ between the set of generic points of maximal dimension of $X$ and that of $X^{\prime}$, and if $\left(\eta_{X}, \sigma\left(\eta_{X}\right)\right)$ is a pair of generic points, then $\kappa\left(\eta_{X}\right) \simeq \kappa\left(\sigma\left(\eta_{X}\right)\right)$ (over $k$ ).

b) By definition, constructing a piecewise isomorphism can be realized by a finite number of steps. Let $X$ and $X^{\prime}$ be two $k$-varieties of the same dimension $d$. Step 1: Construct a $k$-isomorphism $U \simeq U^{\prime}$ on open sets of $X$ and $X^{\prime}$ containing all the points of dimension $d$. Step 2: Apply Step 1 to the $k$-varieties $(X \backslash U)_{\text {red }}$ and $\left(X^{\prime} \backslash U^{\prime}\right)_{\text {red }}$, and so on. Each time, the dimension is decreased by 1 . Then, after exactly $d$ steps, we have constructed the required partitions of $X$ and $X^{\prime}$.

c) We can complete Theorem 2.3 by the following statements (see [7, Lemma 9]), in small dimensions: if $k$ is an algebraically closed field of characteristic zero and if $X, X^{\prime}$ are $k$-curves (surfaces) that are stably birational, then $X$ is birational to 
$X^{\prime}$. Such a result uses crucially the so-called Lüroth problem (i.e., Castelnuovo's criterion in dimension 2).

\section{A NEW ANSWER to LARSEN-LUNTS' QUESTION}

In this section, we complete, for $k$-varieties of dimension one or two, the answer to question LL, which we have given in [7] (see Remark 3.6). In particular, if $k$ is an algebraically closed field of characteristic zero, we link question LL for (uni)ruled surfaces to a condition on the nature of $\mathbb{L}$ in $K_{0}\left(\operatorname{Var}_{k}\right)$.

Lemma 3.1. Let $k$ be a field of characteristic zero. Let $X$ be a k-variety of dimension $d$ and let $\bar{X}$ be a smooth projective model of $X$. Then there exists a finite number of connected smooth projective $k$-varieties $C_{i}, i \in I$, of dimension at most $d-1$, such that

$$
[\bar{X}]-[X]=\sum_{i \in I} \varepsilon_{i}\left[C_{i}\right]
$$

with $\varepsilon_{i} \in\{-1,1\}$ for each $i \in I$.

Proof. The proof comes directly from the relations in $K_{0}\left(\operatorname{Var}_{k}\right)$ and from Hironaka's theorem on the existence of resolutions of singularities.

Proposition 3.2. Let $k$ be a field of characteristic zero and let $n \geq 1$ be an integer. Let $X_{i}, X_{i}^{\prime}, 1 \leq i \leq n$, be irreducible $k$-varieties of dimension d. Assume that there exists a finite number of $k$-varieties $V_{s}, s \in S$, and $W_{t}, t \in T$, of dimension at most $d-1$, such that

$$
\sum_{1 \leq i \leq n}\left[X_{i}\right]-\sum_{1 \leq i \leq n}\left[X_{i}^{\prime}\right]=\sum_{s \in S}\left[V_{s}\right]-\sum_{t \in T}\left[W_{t}\right]
$$

in $K_{0}\left(\operatorname{Var}_{k}\right)$. Assume that one of the following properties holds:

a) for each $i, 1 \leq i \leq n, X_{i}$ is non-uniruled;

b) $k$ is algebraically closed, $d=2$, and, for each $i, 1 \leq i \leq n, X_{i}$ is either non-(uni)ruled or rational;

c) $k$ is algebraically closed, $d=2$, and the kernel of the multiplication by $\mathbb{L}$ in $K_{0}\left(\operatorname{Var}_{k}\right)$ does not contain any (non-trivial) element of dimension 1.

Then there exists a bijection $\sigma:\{1, \ldots, n\} \rightarrow\{1, \ldots, n\}$ such that $X_{i}$ and $X_{\sigma(i)}^{\prime}$ are birational, for each $i, 1 \leq i \leq n$. In particular, under the same assumption, if $\sum_{1 \leq i \leq n}\left[X_{i}\right]=\sum_{1 \leq i \leq n}\left[X_{i}^{\prime}\right]$ in $\bar{K}_{0}\left(\operatorname{Var}_{k}\right)$, then $X_{i}$ is birational to $X_{\sigma(i)}^{\prime}$, for each $i, 1 \leq i \leq n$.

Proof. Thanks to Lemma 3.1, one sees that, at the same time, we can replace each $k$-variety of $(*)$ by a smooth projective model and preserve a relation analogous to $(*)$. Thus, for proving the announced birationality property, we can assume that all the $X_{i}$, all the $X_{i}^{\prime}$, all the $V_{s}$, and all the $W_{t}$ are connected smooth and projective. Now apply $S B$ to the (new) relation $(*)$. Since $\mathbb{Z}[\mathfrak{s} \mathfrak{b}]$ is a free abelian group, one can remark that $|S|=|T|$. Let $i$ be such that $1 \leq i \leq n$. Two cases occur:

i) There exists some $s \in S$, such that $S B\left(X_{i}\right)=S B\left(V_{s}\right)$. In this case, $X_{i}$ is uniruled, by Theorem 2.3 .

ii) There exists some $j, 1 \leq j \leq n$, such that $S B\left(X_{i}\right)=S B\left(X_{j}^{\prime}\right)$.

In the rest of the proof, we are going to study more precisely these two cases with respect to the different assumptions, in order to conclude, up to renumbering, that $X_{i}$ is birational to $X_{i}^{\prime}$, for each $i, 1 \leq i \leq n$. 
a) Since $X_{i}$ is non-uniruled, $S B\left(X_{i}\right)$ cannot be equal to $S B\left(V_{s}\right)$, for any $s \in S$. So $X_{i}$ is stably birational to $X_{j}^{\prime}$, for some $j, 1 \leq j \leq n$, by the definition of $S B$. Since the number of the $X_{i}$ is equal to that of the $X_{j}^{\prime}$, this correspondence induces a bijection and, up to renumbering, each $X_{i}$ is stably birational to $X_{i}^{\prime}$. By Theorem 2.3 and by using again the non-uniruledness of $X_{i}$, we conclude that $X_{i}$ is birational to $X_{i}^{\prime}$, for each $i, 1 \leq i \leq n$.

b) By assumption, we can suppose that there exists an integer $0 \leq n^{\prime} \leq n$ such that, up to renumbering, $X_{i}$ is non-uniruled for each $i, 1 \leq i \leq n^{\prime}$, and that $X_{i}$ is rational for each $i, 1+n^{\prime} \leq i \leq n$. Applying the arguments used in a), we conclude that up to renumbering, $X_{i}$ is birational to $X_{i}^{\prime}$, for each $i, 1 \leq i \leq n^{\prime}$. Thus there exists an open set $U_{i}$ of $X_{i}$, and a dense open set $U_{i}^{\prime}$ of $X_{i}^{\prime}$ such that $\left[U_{i}\right]=\left[U_{i}^{\prime}\right]$ in $K_{0}\left(\operatorname{Var}_{k}\right)$. It follows that $\left[X_{i}\right]-\left[X_{i}^{\prime}\right]=\left[\left(X_{i} \backslash U_{i}\right)_{\text {red }}\right]-\left[\left(X_{i}^{\prime} \backslash U_{i}^{\prime}\right)_{\text {red }}\right]$. By substituting these equalities in $(*)$, the relation becomes:

$$
\sum_{1 \leq i \leq n^{\prime}}\left[X_{i}\right]-\sum_{1 \leq i \leq n^{\prime}}\left[X_{i}^{\prime}\right]=\sum_{s \in S^{\prime}}\left[V_{s}^{\prime}\right]-\sum_{t \in T^{\prime}}\left[W_{t}^{\prime}\right]
$$

where all the $X_{i}$ are rational, all the $X_{i}^{\prime}$ are uniruled, and all the $V_{s}^{\prime}$ and all the $W_{t}^{\prime}$ are connected smooth and projective. Assume that there exists $i, 1 \leq i \leq n^{\prime}$, such that $S B\left(X_{i}\right)=S B\left(X_{j}^{\prime}\right)$, for some $j, 1 \leq j \leq n^{\prime}$. We then conclude by Remark 2.5.) (see also [7, Lemma 9]) that $X_{i}$ is birational to $X_{j}^{\prime}$. Up to renumbering, and by using the arguments above, we obtain a new relation:

$$
\sum_{1 \leq i \leq n^{\prime \prime}}\left[X_{i}\right]-\sum_{1 \leq i \leq n^{\prime \prime}}\left[X_{i}^{\prime}\right]=\sum_{s \in S^{\prime \prime}}\left[V_{s}^{\prime \prime}\right]-\sum_{t \in T^{\prime \prime}}\left[W_{t}^{\prime \prime}\right] .
$$

This time, all the $X_{i}$ are rational, all the $X_{i}^{\prime}$ are uniruled, all the $V_{s}^{\prime}$ and all the $W_{t}^{\prime}$ are connected smooth and projective, of dimension less than or equal to 1 , and, for each $j, 1 \leq j \leq n^{\prime \prime}, S B\left(X_{j}^{\prime}\right) \neq S B\left(X_{i}\right)=1=S B\left(\mathbb{P}_{k}^{2}\right)$, for any $i, 1 \leq i \leq n^{\prime \prime}$. By Remark 2.5k), we can assume that $S^{\prime \prime}=T^{\prime \prime}=\{1, \ldots, n\}$. Let us fix $j, 1 \leq j \leq n^{\prime \prime}$. Let us set $X^{\prime}:=X_{j}$. There exists $t \in T^{\prime \prime}$ such that $S B\left(X^{\prime}\right)=S B\left(W_{t}^{\prime \prime}\right)$. Let us set $W:=W_{t}^{\prime \prime}$. By definition, $X^{\prime}$ is stably birational to $W$, which is a nonrational connected smooth projective curve. By Remark 2.5c), we conclude that $X^{\prime}$ is birational to $W \times{ }_{k} \mathbb{P}_{k}^{1}$. By eliminating indeterminacies from a birational map $X^{\prime} \rightarrow W \times_{k} \mathbb{P}_{k}^{1}$, we remark that there exists an integer $\gamma \in \mathbb{Z}$ such that

$$
\left[X^{\prime}\right]=[W]+\mathbb{L}([W]+\gamma) .
$$

By applying these arguments to each $j, 1 \leq j \leq n^{\prime \prime}$, by substituting the corresponding equalities in $(* * *)$, we find integers $\gamma_{j} \in \mathbb{Z}, 1 \leq j \leq n^{\prime \prime}$, such that the following relation holds in $K_{0}\left(\operatorname{Var}_{k}\right)$ :

$$
\sum_{1 \leq i \leq n^{\prime \prime}}\left[X_{i}\right]=\mathbb{L} \sum_{1 \leq j \leq n^{\prime \prime}}\left[W_{j}^{\prime \prime}\right]+\sum_{1 \leq s \leq n^{\prime \prime}}\left[V_{s}^{\prime \prime}\right]+\mathbb{L} \sum_{1 \leq j \leq n^{\prime \prime}} \gamma_{j} .
$$

Since $P_{\mathbb{A}_{k}^{1}}(u)=u^{2}$, by comparing the terms of degree 3 of the Poincaré polynomials of the right hand side and the left hand side, we find that $2 \sum_{1 \leq j \leq n^{\prime \prime}} g\left(W_{j}^{\prime \prime}\right)=0$. Thus, for each $j, 1 \leq j \leq n^{\prime \prime}, W_{j}^{\prime \prime}$ is a rational $k$-curve. This is a contradiction. It follows that $X_{i}^{\prime}$ is rational, for each $i, 1 \leq i \leq n^{\prime}$. Conclusion: In any case, up to renumbering, $X_{i}$ is birational to $X_{i}^{\prime}$, for all the $i$, and $X$ is birational to $X^{\prime}$.

c) We are going to follow the strategy of the previous cases. By the arguments used in a), we can assume that all the $X_{i}$ and all the $X_{i}^{\prime}$ are uniruled. By Remark 2.5c), we can assume that, for each $i, 1 \leq i \leq n$, there exists an index $s \in S$ 
such that $S B\left(X_{i}\right)=S B\left(V_{s}\right)$; that for each $j, 1 \leq j \leq n$, there exists an index $t \in T$ such that $S B\left(X_{i}^{\prime}\right)=S B\left(W_{t}\right)$; and that $S=T=\{1, \ldots, n\}$. At last, up to renumbering, we can assume also that $S B\left(X_{i}\right)=S B\left(V_{i}\right)$ and $S B\left(X_{i}^{\prime}\right)=S B\left(W_{i}\right)$, for each $i, 1 \leq i \leq n$. As in $\mathrm{b})$, the relation $(*)$ becomes

$$
\mathbb{L}\left(\sum_{1 \leq i \leq n}\left[V_{i}\right]-\sum_{1 \leq i \leq n}\left[W_{i}\right]\right)=\mathbb{L} \gamma
$$

Computing the Poincaré polynomials in this relation and by comparing, this time, the terms of degree 2 , we conclude that $\gamma=0$. So, by assumption,

$$
\sum_{1 \leq i \leq n}\left[V_{i}\right]=\sum_{1 \leq i \leq n}\left[W_{i}\right]
$$

By Theorem 2.4 ), it follows that, up to renumbering, $V_{i}$ is $k$-isomorphic to $W_{i}$. Thus, for each $i, 1 \leq i \leq n, X_{i}$ is birational to $X_{i}^{\prime}$.

Theorem 3.3. Let $k$ be a field of characteristic zero. Let $X, X^{\prime}$ be two k-varieties such that $[X]=\left[X^{\prime}\right]$ in $K_{0}\left(\operatorname{Var}_{k}\right)$.

a) Let $X, X^{\prime}$ be geometrically irreducible $k$-curves. Then $X$ and $X^{\prime}$ are piecewise isomorphic.

b) Assume that $k$ is algebraically closed and that the dimension of $X$ is 2 .

i) If all the irreducible components of $X$ of dimension 2 are either nonuniruled or rational, then $X$ and $X^{\prime}$ are piecewise isomorphic.

ii) If the kernel of the multiplication by $\mathbb{L}$ in $K_{0}\left(\operatorname{Var}_{k}\right)$ does not contain any (non-trivial) element of dimension 1 , then $X$ and $X^{\prime}$ are piecewise isomorphic.

Proof. By Remark 2.5b), in each case, we have only to prove the birationality of $X$ and $X^{\prime}$. We will then conclude by induction, using Theorem 2.3 a) for proving a), and using Theorem $2.3 \mathrm{~b}$ ) for proving $\mathrm{b}$ ).

a) Let us show that $X$ is birational to $X^{\prime}$. Let $\bar{X}$ and $\bar{X}^{\prime}$ be smooth projective models of $X$ and $X^{\prime}$ respectively. There exists a finite number of finite field extensions $k_{i}, i \in I$, and $k_{j}^{\prime}, j \in J$, of $k$ such that

$$
[\bar{X}]-\left[\overline{X^{\prime}}\right]=\sum_{i \in I}\left[x_{i}\right]-\sum_{j \in J}\left[x_{j}^{\prime}\right]
$$

where $x_{i}:=\operatorname{Spec}\left(k_{i}\right)$ and $x_{j}^{\prime}:=\operatorname{Spec}\left(k_{j}^{\prime}\right)$, for all $i, j$. Two cases occur. If $\bar{X}$ is non-uniruled, then, by Proposition 3.2 ) , we conclude that $\bar{X}$ is $k$-isomorphic to $\bar{X}^{\prime}$. Thus $X$ is birational to $X^{\prime}$. If $S B(\bar{X}) \neq S B\left(\bar{X}^{\prime}\right)$, then there exist $i \in I$ and $j \in J$ such that $S B(\bar{X})=S B\left(x_{i}\right)$ and $S B\left(\bar{X}^{\prime}\right)=S B\left(x_{j}^{\prime}\right)$. As $\bar{X}$ and $\bar{X}^{\prime}$ are geometrically irreducible, then $\bar{X} \simeq \mathbb{P}_{k}^{1} \simeq \bar{X}^{\prime}$. So $X$ and $X^{\prime}$ are birational. Lastly, assume that $\bar{X}$ is uniruled and that $S B(\bar{X})=S B\left(\overline{X^{\prime}}\right)$. Then, $|I|=|J|$ and, up to renumbering, $S B\left(x_{i}\right)=S B\left(x_{i}^{\prime}\right)$, for each $i \in I$. It follows that $k_{i} \simeq k_{i}^{\prime}$ and $\left[x_{i}\right]=\left[x_{i}^{\prime}\right]$ for each $i \in I$. Thus $[\bar{X}]=\left[\bar{X}^{\prime}\right]$ in $K_{0}\left(\operatorname{Var}_{k}\right)$. If $\bar{X} \simeq \mathbb{P}_{k}^{1}$, it follows, by Lüroth's problem, that $\bar{X}^{\prime} \simeq \mathbb{P}_{k}^{1}$. If $\bar{X}$ has no rational point, we conclude by [3, Theorem 1.2].

b) Let $X_{i}, 1 \leq i \leq n$, and $X_{i}^{\prime}, 1 \leq i \leq n^{\prime}$, be the irreducible components of dimension 2 of $X$ and $X^{\prime}$ respectively. By Lemma 2.1b), $n=n^{\prime}$. Let us show that $X$ and $X^{\prime}$ are birational. There exists a finite number of $k$-varieties $V_{s}, s \in S$, and $W_{t}, t \in T$, of dimension less than or equal to 1 such that $\sum_{1 \leq i \leq n}\left[X_{i}\right]-$ $\sum_{1 \leq i \leq n}\left[X_{i}^{\prime}\right]=\sum_{s \in S}\left[V_{s}\right]-\sum_{t \in T}\left[W_{t}\right]$. For $\left.i\right)$, we conclude by Proposition $[3.2 b$ ) 
that, up to renumbering, $X_{i}$ is birational to $X_{i}^{\prime}$, for each $i$. So, $X$ is birational to $X^{\prime}$. For $i$ ), we conclude by Proposition $3.2 \mathrm{r}$ ).

Remark 3.4. By the arguments developed in the proof of Theorem 3.3 above, we see that Larsen and Lunts' question can also be formulated in terms of the study of the graded ring associated to the filtration by the dimensions in $K_{0}\left(\operatorname{Var}_{k}\right)$.

Corollary 3.5. Let $k$ be an algebraically closed field of characteristic zero. Let $X$, $X^{\prime}$ be two $k$-surfaces such that $[X]=\left[X^{\prime}\right]$. Assume that $\mathbb{L}$ is not a zero divisor in $K_{0}\left(\operatorname{Var}_{k}\right)$. Then $X$ is piecewise isomorphic to $X^{\prime}$.

Remark 3.6. Up to now, the only progress on question LL was contained in [7, §5], where we have proved that this question admits a positive answer in the following cases:

a) if $k$ is an arbitrary field of characteristic zero, for zero-dimensional $k$-varieties (see [7. §5.1]), or for $k$-varieties $X$ such that $X_{\bar{k}}$ does not contain any irreducible rational curve (here $\bar{k}$ is an algebraic closure of $k$; see [7, §5.4]);

b) if $k$ is an algebraically closed field of characteristic zero, for curves, or for smooth and projective surfaces (see [7, $\S \S 5.2,5.3]$ ), or for $k$-varieties containing only finitely many rational curves (see [7, §5.4]).

In particular, observe that:

$\left.\mathrm{a}^{\prime}\right)$ for a (Severi-Brauer) conic $C, C_{\bar{k}}$ contains (trivially) a rational $\bar{k}$-curve;

$\mathrm{b}^{\prime}$ ) one knows that there exist non-(uni)ruled smooth projective surfaces with infinitely many rational curves. In particular, there exist open subvarieties of such surfaces having the same property.

For these reasons, Theorem 3.3 cannot be deduced from results of $77, \S 5]$.

\section{Geometric problems in the Circle OF LARSEN AND LUNTS' QUESTION}

In this section, we set two new challenging problems, linked to question LL. We also see the consequence of a positive answer to question LL on the (more classical) piecewise cancellation problem.

4.1. Larsen and Lunts' question and rational points on varieties. We ask the following question:

Question R. Let $k$ be a field of characteristic zero and let $m \in \mathbf{N} \backslash\{0\}$. Let $X$ be

a $k$-scheme of finite type. Are the following assertions equivalent?

i) $X$ has $m$ rational points;

ii) there exists a $k$-scheme of finite type $Y$ such that $[X]=[Y]+m$ in $K_{0}\left(\operatorname{Var}_{k}\right)$.

In dimension zero, this is true by Theorem $2.4 \mathrm{k}$ ). Observe also that $i$ ) $\Rightarrow$ ii) (in any characteristic).

Lemma 4.1. Let $k$ be a field of characteristic zero. Let $X$ be a connected smooth proper $k$-variety. Then $X$ has a rational point if and only if there exists a connected smooth proper $k$-variety $\bar{Y}$ and a smooth divisor $D$ on $\bar{Y}$ such that $Y:=\bar{Y} \backslash D$ satisfies the relation $[X]=1+[Y]$ in $K_{0}\left(\operatorname{Var}_{k}\right)$. 
Proof. We have only to prove $\Leftarrow$. More generally, we are going to prove that if there exists an integer $m \geq 1$ such that $[X]=m+[Y]$ (with $Y$ as in the statement), then $X$ has a rational point. Assume that $D:=\sum_{i \in I} D_{i}$. By assumption,

$$
[X]+\sum_{i \in I}\left[D_{i}\right]=m+[\bar{Y}]
$$

Applying $S B$ to this relation, we see that $|I|=m$ (in particular, $D$ is irreducible if and only if $m=1$ ). If $S B(X)=1=S B\left(\mathbb{P}_{k}^{1}\right)$, then $X$ has a rational point. If $S B\left(D_{i}\right)=1$ for some $i$, then $S B(X)=S B(\bar{Y})$ and $D_{i}$ has a rational point. It follows that $\bar{Y}$ also has a rational point. By definition of $S B$, there exists an integer $s$ such that $X \times_{k} \mathbb{P}_{k}^{s}$ is birational to $\bar{Y} \times_{k} \mathbb{P}_{k}^{s}$. As $X \times_{k} \mathbb{P}_{k}^{s}$ and $\bar{Y} \times_{k} \mathbb{P}_{k}^{s}$ are smooth and proper, by the Lang-Nishimura Lemma, the variety $X \times_{k} \mathbb{P}_{k}^{s}$ has a rational point. Thus $X$ has a rational point too.

Proposition 4.2. Let $k$ be a field of characteristic zero and let $m \geq 1$ be an integer. Let $X$ be a geometrically irreducible smooth $k$-curve. Then $X$ has $m$ rational points if and only if there exists a geometrically irreducible smooth $k$-curve $Y$ such that $[X]=m+[Y]$ in $K_{0}\left(\operatorname{Var}_{k}\right)$.

Remark 4.3. Proposition 4.2 implies easily the following consequence: if $X^{\prime}$ is another geometrically irreducible smooth $k$-curve such that $[X]=\left[X^{\prime}\right]$ in $K_{0}\left(\operatorname{Var}_{k}\right)$, then $X$ has $m$ rational points (respectively $m$ effective 0 -cycles of degree $d$ ) if and only if $X^{\prime}$ has $m$ rational points (respectively $m$ effective 0-cycles of degree $d$ ).

Proof. We only have to show $\Leftarrow$. We are going to show it by induction on $m$.

a) Assume first that $m=1$. Let $\bar{X}$ (respectively $\bar{Y}$ ) be a smooth projective model of $X$ (respectively $Y$ ). There exists a finite number of finite field extensions $k_{i}, i \in I$, and $k_{j}^{\prime}, j \in J$, of $k$ such that

$$
[\bar{X}]-\sum_{i \in I}\left[x_{i}\right]=1+[\bar{Y}]-\sum_{j \in J}\left[x_{j}^{\prime}\right]
$$

with $x_{i}:=\operatorname{Spec}\left(k_{i}\right)$, for $i \in I$, and $x_{j}^{\prime}:=\operatorname{Spec}\left(k_{j}^{\prime}\right)$, for $j \in J$. Applying $S B$ to this relation, remark first that $|J|=|I|+1$. Besides, two cases occur. In the first one, $S B(\bar{X})=S B\left(\mathbb{P}_{k}^{1}\right)=1$ or $S B(\bar{X})=S B\left(x_{i}\right)$, for some $i \in I$. Since $\bar{X}$ is geometrically irreducible, it follows that $\bar{X} \simeq \mathbb{P}_{k}^{1}$, and $X$ has a rational point. In the second one, $\bar{X}, \bar{Y}$ are stably birational, and $S B\left(x_{j}^{\prime}\right)=S B(\operatorname{Spec}(k))=1$, for some $j \in J$. So $x_{j}^{\prime} \in \bar{Y}(k)$ and $\bar{X}$ has a rational point, say $x \in \bar{X}(k)$. If $x \notin X(k)$, necessarily $x=x_{s}$, for some $s \in I$, and we can write:

$$
[X]=[\bar{X}]-1-\sum_{i \in I, x_{i} \neq x}\left[x_{i}\right]=1+[Y]=[\bar{Y}]-\sum_{\ell \in J \backslash\{j\}}\left[x_{\ell}^{\prime}\right] .
$$

We conclude by induction on $|I|$, using Lemma 4.1 for the initialization, when $|I|=0$.

b) Assume that $m \geq 1$. If there exists a smooth geometrically irreducible $k$-curve such that $[X]=(1+m)+[Y]$, then, by the arguments that we have developed in a) (use the proof of Lemma 4.1 for the initialization), we conclude that $X$ has a rational point, say $x \in X(k)$. Since $X \backslash\{x\}$ is also a geometrically irreducible smooth $k$-curve such that $[X \backslash\{x\}]=m+[Y]$ in $K_{0}\left(\operatorname{Var}_{k}\right)$, we conclude, by the induction hypothesis, that $X \backslash\{x\}$ has $m$ rational points. So $X$ has $m+1$ rational points. The result follows. 
4.2. Larsen and Lunts' question and a piecewise analog of the "complement problem". Let $N \geq 1$ be an integer. Let $f, g \in \mathbb{C}[\underline{x}]:=\mathbb{C}\left[x_{1}, \ldots, x_{N}\right]$ be two irreducible polynomials. Assume that there exists a $\mathbb{C}$-isomorphism $\mathbb{C}[\underline{x}]_{f} \rightarrow$ $\mathbb{C}[\underline{x}]_{g}$. The complement problem can be expressed by the following question: Is it true that the hypersurfaces $V(f)$ and $V(g)$ of $\mathbb{A}_{\mathbb{C}}^{N}$ are $\mathbb{C}$-isomorphic? One knows no complete answer to this classical question of affine geometry, even in dimension one (see 4 4 ). We introduce the following piecewise analog.

Remark 4.4. In a sense, the piecewise analog below is more natural. When $N=2$, if $f, g$ are not assumed to be irreducible, it is easy to construct counterexamples to the complement problem. Yet, in this situation, our piecewise analog is true.

Question Cr. Assume that $k$ is a field of characteristic zero. Let $N$ be an integer. Assume that $\varphi: \mathbb{P}_{k}^{N} \rightarrow \mathbb{P}_{k}^{N}$ is a birational map. Can $\varphi$ be extended in a piecewise isomorphism?

Let $N \geq 2$ be an integer and let $\varphi: \mathbb{P}_{k}^{N} \rightarrow \mathbb{P}_{k}^{N}$ be a birational map. There exist open sets $U, V$ of $\mathbb{P}_{k}^{N}$ such that $\varphi$ induces a $k$-isomorphism $U \simeq V$. Below, we are interested in extending the (fixed) $k$-isomorphism $\varphi: U \rightarrow V$ in a piecewise automorphism of $\mathbb{P}_{k}^{N}$.

Lemma 4.5. Let $k$ be a field of characteristic zero and let $n \geq 1$ be an integer. With the notation above, assume that $\mathbb{P}_{k}^{N} \backslash U$ is of dimension $r \in \mathbb{N}$ and that all the irreducible components $X_{i}$ of dimension $r, 1 \leq i \leq n$, of $\left(\mathbb{P}_{k}^{N} \backslash U\right)_{\text {red }}$ are nonuniruled. Let $X_{i}^{\prime}, 1 \leq i \leq n$, be the reduced irreducible components of dimension $r$ of $\left(\mathbb{P}_{k}^{N} \backslash V\right)_{\text {red }}$. Then there exists a bijection $\sigma:\{1, \ldots, n\} \rightarrow\{1, \ldots, n\}$ such that, for all $i, 1 \leq i \leq n, X_{i}$ is birational to $X_{\sigma(i)}^{\prime}$.

Proof. Remark that $\left[\left(\mathbb{P}_{k}^{N} \backslash U\right)_{\text {red }}\right]=\left[\mathbb{P}_{k}^{N} \backslash U\right]=\left[\mathbb{P}_{k}^{N}\right]-[U]=\left[\mathbb{P}_{k}^{N} \backslash V\right]$ in $K_{0}\left(\operatorname{Var}_{k}\right)$. Thus $\left(\mathbb{P}_{k}^{N} \backslash U\right)_{\text {red }}$ and $\left(\mathbb{P}_{k}^{N} \backslash V\right)_{\text {red }}$ have the same dimension $r$ and the same number $n$ of irreducible components of dimension $r$. There exists a finite number of $k$-varieties $V_{s}, s \in S$, and $W_{t}, t \in T$, of dimension less than and or equal to $r-1$ such that

$$
\sum_{1 \leq i \leq n}\left[X_{i}\right]-\sum_{1 \leq i \leq n}\left[X_{i}^{\prime}\right]=\sum_{s \in S}\left[V_{s}\right]-\sum_{t \in T}\left[W_{t}\right]
$$

in $K_{0}\left(\operatorname{Var}_{k}\right)$. We conclude by Proposition $\left.3.2 \mathrm{a}\right)$.

Proposition 4.6. Let $k$ be an algebraically closed field of characteristic zero. Let $N \geq 1$ be an integer and let $\varphi: \mathbb{P}_{k}^{N} \rightarrow \mathbb{P}_{k}^{N}$ be a birational map.

a) Assume that $N \leq 2$. Then $\varphi$ can be extended to a piecewise isomorphism.

b) Assume that $N \geq 3$. With the notation above, if $\mathbb{P}_{k}^{N} \backslash U$ is of dimension $r \leq 1$ or if $r=2$ and if the reduced irreducible components of dimension 2 of $\left(\mathbb{P}_{k}^{\bar{N}} \backslash U\right)_{\text {red }}$ are either non-uniruled or rational, then $\varphi$ can be extended in a piecewise isomorphism.

Proof. For a), this comes directly from Theorem 2.4b); for b), it comes directly from Theorem $3.3 \mathrm{~b}$ ) $i$ ). 
4.3. Larsen and Lunts' question and a piecewise analog of Zariski's cancellation problem. If $X, X^{\prime}$ are $k$-varieties such that $X \times_{k} \mathbb{A}_{k}^{1} \simeq X^{\prime} \times_{k} \mathbb{A}_{k}^{1}$, is it true that $X \simeq X^{\prime}$ (over $k$ )? This is the so-called Zariski cancellation problem (which is not true in general). Motivic integration has suggested the following piecewise analog. Recall that an element $a$ of a ring $A$ is said to be regular if it is not a zero divisor in $A$.

Remark 4.7. The known counterexamples of Zariski's cancellation problem, such as Danielewski's varieties, which are piecewise isomorphic, give no information for solving the problem stated in question $\mathrm{Z}$ below. We ask a more refined question: Is it possible to find two irreducible, not birational, $k$-varieties $X$ and $X^{\prime}$ such that $X \times_{k} \mathbb{A}_{k}^{1} \simeq X^{\prime} \times{ }_{k} \mathbb{A}_{k}^{1}$ ? Up to now, this last question is open.

Question Z. Assume that $k$ is a field of characteristic zero. Is $\mathbb{L}$ a regular element in $K_{0}\left(\operatorname{Var}_{k}\right)$ ?

Lemma 4.8. Let $k$ be a field of characteristic zero. Let $X_{i}, 1 \leq i \leq n, X_{j}^{\prime}$, $1 \leq j \leq m, V_{s}, s \in S$, and $W_{t}, t \in T$, be $k$-varieties of dimensiond such that

$$
\mathbb{L}\left(\sum_{1 \leq i \leq n}\left[X_{i}\right]-\sum_{1 \leq j \leq m}\left[X_{j}^{\prime}\right]\right)=\sum_{s \in S}\left[V_{s}\right]-\sum_{t \in T}\left[W_{t}\right]
$$

in $K_{0}\left(\operatorname{Var}_{k}\right)$. Assume that question $L L$ admits a positive answer for all the quasiprojective $k$-varieties of dimension less than or equal to $d+1$. Then the number of irreducible components of dimension d of all the $X_{i}$ is equal to that of the irreducible components of dimension $d$ of all the $X_{j}^{\prime}$. Besides, for each $i, 1 \leq i \leq n$, each irreducible component of dimension $d$ of $X_{i}$ is stably birational to an irreducible component of dimension d of some $X_{j}$, for $j, 1 \leq j \leq m$.

Proof. Let us denote by $\left(X_{i, \alpha}\right)_{\alpha}$ the irreducible components of dimension $d$ of $X_{i}$, and by $\left(X_{j, \beta}^{\prime}\right)_{\beta}$ the irreducible components of dimension $d$ of $X_{j}^{\prime}$. There exist $k$ varieties $V_{s}^{\prime}, s \in S^{\prime}, W_{t}^{\prime}, t \in T^{\prime}$, of dimension $d-1$ such that

$$
\left[Z:=\left(\bigsqcup_{i, \alpha} X_{i, \alpha} \times_{k} \mathbb{A}_{k}^{1}\right) \sqcup\left(\bigsqcup_{s} V_{s}^{\prime}\right)\right]=\left[\left(\bigsqcup_{j, \beta} X_{j, \beta}^{\prime} \times_{k} \mathbb{A}_{k}^{1}\right) \sqcup\left(\bigsqcup_{t} W_{t}^{\prime}\right)=: Z^{\prime}\right] .
$$

By assumption, $Z$ and $Z^{\prime}$ are of dimension $d+1$. The assertions come directly from Lemma 2.1 and Remark 2.5 $\mathrm{k}$ ).

Proposition 4.9. Let $k$ be an algebraically closed field of characteristic zero and let $r \leq 3$ be an integer. Assume that question LL admits a positive answer for all the quasi-projective $k$-varieties of dimension less than or equal to $r$. Then the kernel of the multiplication by $\mathbb{L}$ in $K_{0}\left(V_{a r}\right)$ has no (non-trivial) element of dimension less than or equal to $r-1$.

Notice that this property is unconditional for $r=1$. Let $\mathfrak{a} \in K_{0}\left(\operatorname{Var}_{k}\right)$ be an element of dimension 0. Then there exists an integer $m$ such that $\mathfrak{a}=m$ in $K_{0}\left(\operatorname{Var}_{k}\right)$. If $m \mathbb{L}=0$, by computing the Poincaré polynomials in this relation, we conclude that $m u^{2}=0$ in $\mathbb{Z}[u]$. Thus $m=0$.

Proof. Let $\mathfrak{a} \in K_{0}\left(\operatorname{Var}_{k}\right)$ of dimension $r-1$ such that $\mathfrak{a} \mathbb{L}=0$ in $K_{0}\left(\operatorname{Var}_{k}\right)$. There exists a finite number of connected smooth projective $k$-varieties $X_{i}, i \in I$, and $Y_{j}$, 
$j \in J$, of dimension less than or equal to $r-1$ such that $\mathfrak{a}=\sum_{i \in I}\left[X_{i}\right]-\sum_{j \in J}\left[Y_{j}\right]$ and

$$
\mathbb{L} \sum_{i \in I}\left[X_{i}\right]=\mathbb{L} \sum_{j \in J}\left[Y_{j}\right]
$$

Let us show that $\left[\bigsqcup_{i \in I} X_{i}\right]=\sum_{i \in I}\left[X_{i}\right]=\sum_{j \in J}\left[Y_{j}\right]=\left[\bigsqcup_{j \in J} Y_{j}\right]$. The strategy is to prove that $\bigsqcup_{i \in I} X_{i}$ is piecewise isomorphic to $\bigsqcup_{j \in J} X_{j}$. By Lemma 4.8, we see that there exists an integer $n \geq 1$ such that the family of the $X_{i}$ and the family of the $Y_{j}$ contain the same number $n$ of $k$-varieties of maximal dimension (it can also be seen unconditionally by computing Poincaré polynomials). We can assume that $X_{1}, \ldots, X_{n}$ and $Y_{1}, \ldots, Y_{n}$ are these $k$-varieties. Besides, by Lemma 4.8, up to renumbering, for each $i, 1 \leq i \leq n, X_{i} \times{ }_{k} \mathbb{A}_{k}^{1}$ is birational to some $Y_{i} \times{ }_{k} \mathbb{A}_{k}^{1}$.

a) Assume that $r=2$. By Remark $2.5 \mathrm{k}$ ), we conclude that there exist integers $m, \ell \in \mathbb{N}$ such that

$$
\bigsqcup_{i \in I} X_{i} \simeq\left(\bigsqcup_{i=1}^{m} \operatorname{Spec}(k)\right) \sqcup\left(\bigsqcup_{i=1}^{n-\ell} \mathbb{P}_{k}^{1}\right) \sqcup\left(\bigsqcup_{i=1}^{\ell} X_{i}\right) \simeq \bigsqcup_{j \in J} Y_{j} .
$$

b) Assume that $r=3$. Again, by Remark 2.55) (see also [7, Lemma 9]), we conclude that $X_{i}$ is birational to $Y_{i}$, for each $i$. Thus, for each $i, 1 \leq i \leq n$, there exist open sets $U_{i}$ of $X_{i}$ and $V_{i}$ of $Y_{i}$ such that $U_{i} \simeq V_{i}$ (over $k$ ). Let us set $C_{i}:=X_{i} \backslash U_{i}$ and $D_{i}:=Y_{i} \backslash V_{i}$. We can assume that all the $C_{i}$ and all the $D_{i}$ are reduced. So, the relation $(\diamond)$ takes the following form:

$$
\mathbb{L}\left(m_{1}+\sum_{i \in I^{\prime}}\left[C_{i}^{\prime}\right]+\sum_{1 \leq i \leq n}\left[C_{i}\right]\right)=\mathbb{L}\left(m_{1}+\sum_{j \in J^{\prime}}\left[D_{j}^{\prime}\right]+\sum_{1 \leq i \leq n}\left[D_{i}\right]\right),
$$

where $m_{1}, m_{2} \in \mathbb{N}$; the $C_{i}^{\prime}$, for $i \in I^{\prime}$, and the $D_{j}^{\prime}, j \in J^{\prime}$ are connected smooth projective $k$-curves; and the $C_{i}$ and the $D_{i}$, for $1 \leq i \leq n$, are projective $k$ varieties of dimension less than or equal to 1 . Following Remark 2.5b), we have to prove that $Z:=\left(\bigsqcup_{i \in I^{\prime}} C_{i}^{\prime}\right) \sqcup\left(\bigsqcup_{1 \leq i \leq n} C_{i}\right)$ is birational to $\left(\bigsqcup_{j \in J^{\prime}} D_{i}^{\prime}\right) \sqcup\left(\bigsqcup_{1 \leq i \leq n} D_{i}\right)$. The result then follows, by Lemma 4.8 and Remark $2.5 \mathrm{c})$. The relation $(\diamond)$ can be reduced now to a relation with components of dimension zero. We conclude by computing Poincaré polynomials. By Remark $2.5 \mathrm{~b}$ ), we have constructed the required piecewise isomorphism.

Remark 4.10. The strategy, used in the proof of Proposition 4.9 consists in simplifying, "birationally", by $\mathbf{A}_{k}^{1}$. So we can extend the result of Proposition 4.9 by induction to other classes of varieties (or elements of $K_{0}\left(\operatorname{Var}_{k}\right)$ ) as soon as we know how to simplify.

Remark 4.11. The statement for $r=2$ and Theorem 3.3 imply that if $k$ is an algebraically closed field of characteristic zero, the $k$-surfaces satisfy the condition of question LL if and only if the kernel of the multiplication by $\mathbb{L}$ in $K_{0}\left(\operatorname{Var}_{k}\right)$ does not contain any (non-trivial) element of dimension 1.

\section{REFERENCES}

1. Bittner, Franziska. The universal Euler characteristic for varieties of characteristic zero, Compos. Math. 140 (2004), no. 4, 1011-1032. MR2059227(2005d:14031)

2. Denef, Jan; Loeser, François. On some rational generating series occurring in arithmetic geometry. Geometric aspects of Dwork theory. Vols. I, II, 509-526, Walter de Gruyter, Berlin, 2004. MR2099079 (2005h:11267) 
3. Hogadi, Amit. Products of Brauer-Severi surfaces. Proc. Amer. Math. Soc. 137 (2009), no. 1, 45-50. MR2439423 (2009g:14021)

4. Kraft, Hanspeter. Challenging problems on affine $n$-space. Séminaire Bourbaki, Vol. 1994/95. Astérisque No. 237 (1996), Exp. No. 802, 5, 295-317. MR1423629 (97m:14042)

5. Larsen, Michael; Lunts, Valery A. Motivic measures and stable birational geometry. Mosc. Math. J. 3 (2003), no. 1, 85-95. MR1996804 (2005a:14026)

6. Liu, Qing. Algebraic geometry and arithmetic curves. Oxford Graduate Texts in Mathematics, 6, Oxford Science Publications, Oxford University Press, Oxford, 2002. MR1917232 (2003g:14001)

7. Liu, Qing; Sebag, Julien. The Grothendieck ring of varieties and piecewise isomorphisms, to appear in Math. Z.

8. Sebag, Julien. Intégration motivique sur les schémas formels. Bull. Soc. Math. France 132 (2004), no. 1, 1-54. MR2075915 (2005e:14017)

Institut de Mathématiques de Bordeaux, Université Bordeaux 1, 351 Cours de la Libération, 33405 Talence Cedex, France

Current address: Institut de Formation et de Recherche Mathématiques and Institut de Recherche Mathématiques de Rennes, 263 Avenue du Général Leclerc, CS 74205, 35042 Rennes cedex, France

E-mail address: julien.sebag@univ-rennes1.fr 